\title{
INVENTARIO DE LAS ARAÑAS (ARACHNIDA: ARANEAE) DEL PARQUE NACIONAL SIERRA MARTÍN GARCÍA, REPÚBLICA DOMINICANA, CON NUEVOS REGISTROS DE GÉNERO Y ESPECIE PARA LA HISPANIOLA
}

\author{
Gabriel de los Santos y Solanlly Carrero Jiménez \\ Museo Nacional de Historia Natural "Prof. Eugenio de Jesús Marcano". Calle César Nicolás Penson, \\ Plaza de la Cultura Juan Pablo Duarte, Santo Domingo, República Dominicana. \\ g.delossantos@mnhn.gov.do; s.carrero@mnhn.gov.do
}

\section{RESUMEN}

Se ofrecen los resultados de un inventario de las arañas del Parque Nacional Sierra Martín García, República Dominicana. Se registran 90 especies, 79 géneros y 33 familias para esta área protegida. El género Fageicera Dumitrescu et Georgescu, 1992 (Ochyrocerathidae) y la especie Yabisi habanensis Rheims et Brescovit, 2004 (Hersiilidae) se registran por primera vez para la República Dominicana y la Hispaniola. Se consideran como objetos de conservación a las 16 especies endémicas, particularmente Aculepeira busu Levi, 1991, Bythocrotus crypticus Zhang et Maddison, 2012 y Thaloe remotus (Bryant, 1948); las dos primeras por ser endemismos locales de este parque y la tercera por ser una especie muy rara, siendo esta apenas la segunda localidad donde se registra.

Palabras clave: inventario, arañas, Sierra Martín García, República Dominicana, Hispaniola.

\section{ABSTRACT}

The results of an inventory of the spiders from Sierra Martín García National Park, Dominican Republic are presented. A total of 90 species, 79 genera and 33 families are registered from this protected area. The genus Fageicera Dumitrescu et Georgescu, 1992 (Ochyrocerathidae) and the species Yabisi habanensis Rheims et Brescovit, 2004 (Hersiilidae) are recorded for the first time for the Dominican Republic and Hispaniola. Sixteen endemic species are considered as conservation targets for this park, particularly Aculepeira busu Levi, 1991, Bythocrotus crypticus Zhang et Maddison, 2012 and Thaloe remotus (Bryant, 1948); the first two because are local endemisms of this protected area; and the third is a very rare species, known only from a single locality.

Keywords: inventory, spiders, Sierra Martín García, Dominican Republic, Hispaniola.

El Parque Nacional Sierra Martín García, con una extensión de 268 km² (Ley 202-2004: 24-25), se encuentra ubicado entre las provincias de Azua y Barahona, en la parte oriental de la Bahía de Neiba, en el suroeste de la República Dominicana. Su parte más alta alcanza los 1,350 m en la loma El Curro o Alto de la Bandera y sus límites incluyen zonas costeras en su parte sur. De acuerdo con García et al. (2007), entre los tipos de vegetación que se encuentran en esta Sierra están: el monte espinoso subtropical, el bosque seco, el bosque de transición de seco a húmedo, el bosque húmedo, el bosque nublado y una vegetación ribereña asociada a las diferentes cañadas que se encuentran en el área. 
La "Evaluación de los recursos naturales en la Sierra Martín García y Bahía de Neiba" (SEA/DED, 1990) es el trabajo más amplio sobre la fauna de este Parque Nacional al que se puede hacer referencia, aunque la fauna de artrópodos no fue incluida. Particularmente las arañas de esta área protegida han recibido poca atención. Los únicos registros publicados corresponden a las especies endémicas Aculepeira busu Levi, 1991 (Araneidae), Bythocrotus crypticus Zhang et Maddison, 2012 (Salticidae) y Nops hispaniola Sánchez-Ruiz, Brescovit et Alayón, 2015 (Caponiidae); encontrándose en el parque las localidades tipo de las dos primeras.

Recientemente se realizaron tres expediciones al Parque Nacional Sierra Martín García, coordinadas por investigadores del Museo Nacional de Historia Natural "Profesor Eugenio de Jesús Marcano" (MNHNSD) con el objetivo de hacer un inventario de la fauna del área. Estas expediciones tuvieron lugar del 31 de julio al 04 de agosto de 2013; del 05 al 08 de septiembre del mismo año y del 30 de mayo al 01 de junio de 2014. Además de las arañas, fueron estudiados los anfibios, los reptiles y las aves (resultados aún sin publicar), y dentro de los insectos, las mariposas diurnas (Rodríguez et al., 2014).

Atendiendo al tipo de vegetación se seleccionaron los siguientes puntos (Fig. 1): 1. Bosque

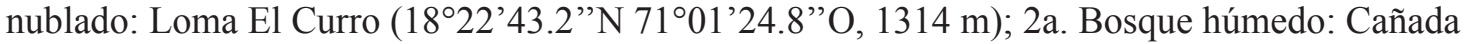

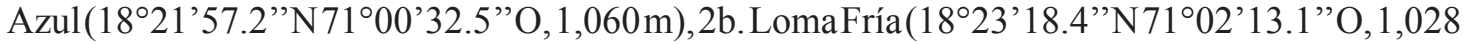
$\mathrm{m})$; 3. Bosque de transición de seco a húmedo: Montilla Arriba $\left(18^{\circ} 23\right.$ '42.0' $\mathrm{N} 71^{\circ} 02$ '23.5' $\mathrm{O}$, $894 \mathrm{~m})$; 4. Bosque seco subtropical: Montilla Abajo (18²3'50.8'” 7104'45.8' O, $419 \mathrm{~m}) ; 5$.

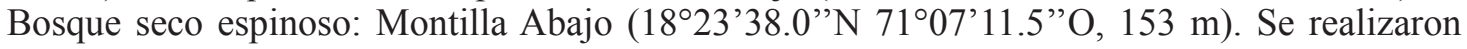
colectas libres diurnas entre las 09:00 y las 16:30 horas, en todos los puntos, y nocturnas entre las 20:00 y las 00:00 horas, con excepción de Cañada Azul. Los especímenes se colectaron en alcohol etílico al 70\% y parte de estos se depositaron en la Colección de Arácnidos del MNHNSD.

Se registran 90 especies de arañas para el Parque Nacional Sierra Martín García, representando este número el $21 \%$ de las especies registradas para la Hispaniola (República Dominicana y Haití); las mismas se distribuyen en 79 géneros y 33 familias. De estas especies, 16 son endémicas de la Hispaniola, distinguiéndose las endémicas locales Aculepeira busu Levi, 1991 y Bythocrotus crypticus Zhang et Maddison, 2012. Excepto las endémicas locales y Nops hispaniola Sánchez-Ruiz, Brescovit et Alayón, 2015, el resto de las especies listadas en este trabajo representan nuevos registros para esta área protegida. Algunos de estos registros se destacan, como es el caso del anyphaenido especie tipo del género Thaloe Brescovit: Thaloe remotus (Bryant, 1948). Esta especie es considerada muy rara, ya que solo se conocía del holotipo colectado en 1938, en el Pico Diego de Ocampo, Prov. Santiago, República Dominicana (Byrant, 1948; Brescovit, 1993). Además, en este trabajo se registran por primera vez para la República Dominicana y la Hispaniola el género Fageicera Dumitrescu et Georgescu, 1992 (Ochyrocerathidae) y la especie Yabisi habanensis Rheims et Brescovit, 2004 (Hersiilidae; Fig. 2). El género Fageicera solo se conoce de tres especies endémicas de Cuba (Dumitrescu et Georgescu, 1992; World Spider Catalog, 2015), los especímenes colectados en Hispaniola no han sido identificados a nivel específico, pero debido a la cercanía geográfica de ambas islas podrían pertenecer a alguna de las especies descritas. Por su parte, $Y$. habanensis es conocida de Cuba y del estado de Florida en EUA (Rheims y Brescovit, 2004). De esta especie se colectó un macho y una hembra (colgando de sus respectivos hilos de seda) durante la última expedición luego de una intensa lluvia, pasadas las 23:00 horas. Otro registro que se destaca son los especímenes pertenecientes al género Syspira (Miturgidae), los cuales no se corresponden con la especie S. eclectica Chamberlin, 1924 registrada para la República Dominicana por Ramírez (2014). Los nuevos registros listados en este trabajo aumentan el número de géneros y especies para la Hispaniola a 199 y 416, respectivamente. 


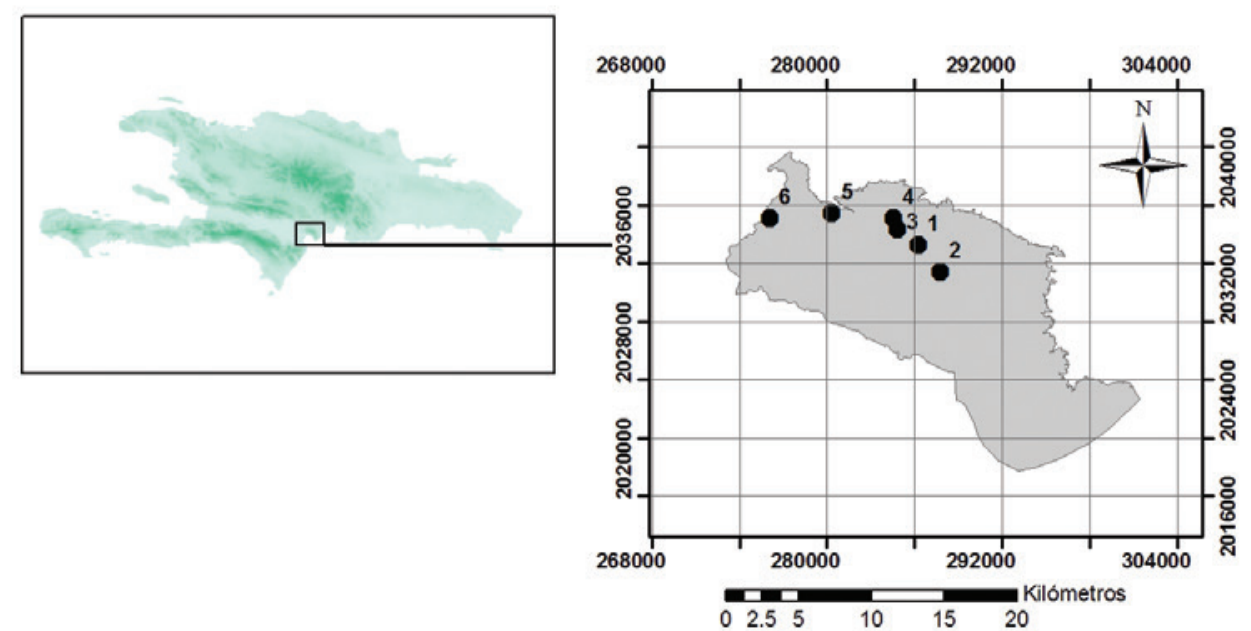

Figura 1. Sitios de muestreo dentro del Parque Nacional Sierra Martín García. 1. Bosque nublado: Loma El Curro; 2a. Bosque húmedo: Cañada Azul, 2b. Loma Fría; 3. Bosque de transición de seco a húmedo: Montilla Arriba; 4. Bosque seco subtropical: Montilla Abajo; 5. Bosque seco espinoso: Montilla Abajo.

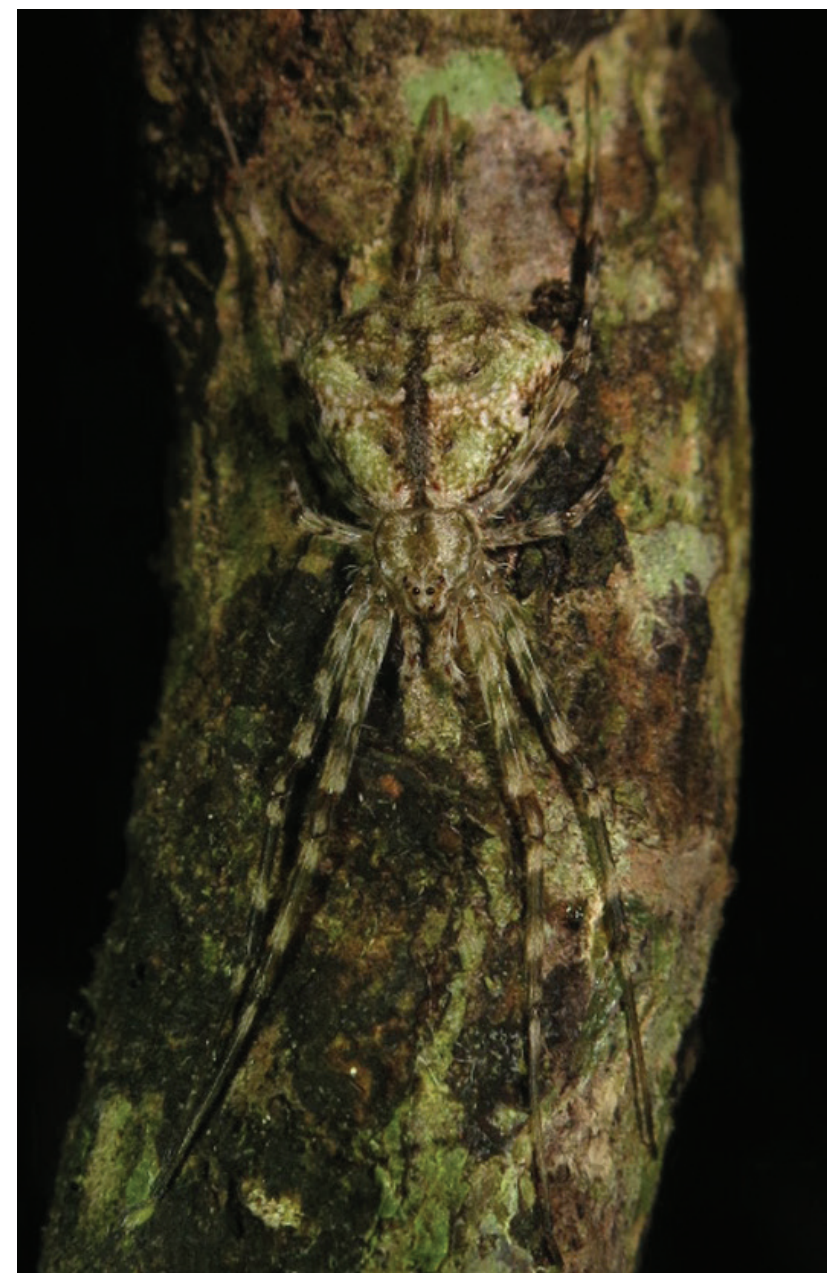

Figura 2. Hembra de Yabisi habanensis Rheims et Brescovit, 2004. Fotografiada en el bosque nublado, Loma El Curro. 
Como objetos de conservación de la fauna de arañas del Parque Nacional Sierra Martín García se eligieron las 16 especies endémicas listadas, particularmente Aculepeira busu, Bythocrotus crypticus y Thaloe remotus; las dos primeras por ser endemismos locales de este parque y la tercera por ser una especie muy rara, siendo esta apenas la segunda localidad donde se registra.

\section{AGRADECIMIENTOS}

Al equipo del Museo Nacional de Historia Natural "Profesor Eugenio de Jesús Marcano" que nos acompañó en las expediciones y de igual forma a los guías Sebastián de la Paz Méndez "Nora" y Bertho Díaz "Veterano" por su eficiente servicio. A Cristina Rheims, Instituto Butantan, Brasil, por la identificación de los ejemplares de Yabisi habanensis. Alexander Sánchez-Ruiz, Instituto Butantan, Brasil, hizo importantes correcciones y sugerencias al manuscrito.

\section{LITERATURA CITADA}

Brescovit, A. D. 1993. Thaloe e Bromelina, novos gêneros de aranhas neotropicais da família Anyphaenidae (Arachnida, Araneae). Revista Brasileira de Entomologia, 37: 693-703.

Bryant, E. B. 1948. The spiders of Hispaniola. Bulletin of the Museum of Comparative Zoology at Harvard College, 100: 329-447.

Dumitrescu, M. y M. Georgescu. 1992. Ochyroceratides de Cuba (Araneae). Mémoires de Biospéologie, 19: 143-153.

García, R., B. Peguero, T. Clase, A. Veloz, F. Jiménez y M. Mejía. 2007. Flora y vegetación de las zonas áridas de la Sierra Martín García, República Dominicana. Moscosa, 15: 5-60.

Huber, B. A. y J., Astrin. 2009. Increased sampling blurs morphological and molecular species limits: revision of the Hispaniolan endemic spider genus Tainonia (Araneae: Pholcidae). Invertebrate Systematics, 23: 281-300.

Ramírez, M. J. 2014. The morphology and phylogeny of dionychan spiders (Araneae: Araneomorphae). Bulletin of the American Museum of Natural History, 390: 1-374.

Rheims, C. A. y A. D. Brescovit. 2004. Revision and cladistic analysis of the spider family Hersiliidae (Arachnida, Araneae) with emphasis on Neotropical and Nearctic species. Insect Systematics \& Evolution, 35: 189-239.

Rodríguez, K., F. Paz y R. H. Bastardo. 2014. Diversidad y patrones de distribución de las mariposas diurnas en un gradiente altitudinal del Parque Nacional Sierra Martín García, República Dominicana. Novitates Caribaea, 7: 72-82.

Sánchez-Ruiz, A., A. D. Brescovit y G. Alayón. 2015. Four new caponiids species (Araneae, Caponiidae) from the West Indies and redescription of Nops blandus (Bryant). Zootaxa, 3972 (1): 43-64. doi:10.11646/zootaxa.3972.1.3

SEA/DED. 1990. Evaluación de los recursos naturales en la Sierra Martín García y Bahía de Neiba. Secretaría de Estado de Agricultura y Servicio Alemán de Cooperación SocialTécnica. Santo Domingo, República Dominicana. 80 pp. 
Secretaría de Estado de Medio Ambiente y Recursos Naturales. 2004. Ley Sectorial de Áreas Protegidas, No. 202-04. Santo Domingo, República Dominicana.

World Spider Catalog. 2015. Natural History Museum Bern, online at http://wsc.nmbe.ch, version 16.5, accedido el 25 de julio, 2015.

Zhang, J. y W. Maddison. 2012. New euophryine jumping spiders from the Dominican Republic and Puerto Rico (Araneae: Salticidae: Euophryinae). Zootaxa, 3476: 1-54.

\section{LISTA DE ESPECIES DEL PARQUE NACIONAL SIERRA MARTÍN GARCÍA}

* = endémica de la Hispaniola; ** = nuevo registro para la República Dominicana y la Hispaniola. $* / *$ = considerada endémica por ser un género endémico (Hubber y Astrin, 2009; Zhang y Maddison, 2012; World Spider Catalog, 2015). N, bosque nublado; H, bosque húmedo; BT, bosque de transición de seco a húmedo; S, bosque seco; E, bosque seco espinoso.

\section{INFRAORDEN MYGALOMORPHAE}

\section{DIPLURIDAE}

1. Ischnothele $\mathrm{sp} . \mathrm{S}$

2. Masteria sp. : N, H

CYRTAUCHENIIDAE

3. Bolostromus sp. : H, S

BARYCHELIDAE

4. Psalistops sp. : S, E

THERAPHOSIDAE

5. Avicularia sp. : BT

6. Phormictopus sp. : H, BT, S, E

\section{INFRAORDEN ARANEOMORPHAE}

\section{SICARIIDAE}

7. Loxosceles sp. : S, E

\section{SCYTODIDAE}

8. Scytodes fusca Walckenaer, 1837 : N, S, E

OCHYROCERATHIDAE

9. Fageicera sp.** : H

10. Ochyrocera sp. : N

\section{PHOLCIDAE}

11. Micropholcus sp. : N, H, BT, S

12. Modisimus vittatus Bryant, $1948^{*}$ : S, E

13. Modisimus sp. 1 : N, H, S

14. Modisimus sp. $2: \mathrm{N}, \mathrm{H}$

15. Tainonia sp.*/* N, H, BT, S

CAPONIIDAE

16. Nops hispaniola Sánchez-Ruiz, Brescovit et Alayón, 2015* : N, E

17. Nops gertschi Chickering, 1967* : E 


\section{OONOPIDAE}

18. Heteroonops sp. : $\mathrm{H}$

19. Scaphiella sp. : S

20. Stenoonops sp. : N

\section{MIMETIDAE}

21. Mimetus sp. : H, BT

\section{HERSIILIDAE}

22. Yabisi habanensis Rheims et Brescovit, 2004** : N, H

\section{DEINOPIDAE}

23. Deinopis lamia MacLeay, 1839 : N, H, S, E

\section{ULOBORIDAE}

24. Miagrammopes sp. : H, S

25. Philoponella sp. : S

26. Uloborus sp. : S

\section{THERIDIIDAE}

27. Anelosimus sp. : N, H, S

28. Dipoena sp. : N, H, BT, S

29. Latrodectus geometricus C. L. Koch, 1841 : N

30. Latrodectus mactans (Fabricius, 1775) : N, S

31. Neopisinus gratiosus (Bryant, 1940) : N, BT

32. Spintharus flavidus Hentz, 1850 : N, H

33. Steatoda sp. : N, H

34. Rhomphaea fictilium (Hentz, 1850) : S

35. Theridion $\mathrm{sp}$ : : $\mathrm{H}$

36. Thymoites sp. : N

\section{LINYPHIIDAE}

37. Florinda sp. : N, BT

38. Frontinella $\mathrm{sp}$. : S

\section{NEPHILIDAE}

39. Nephila clavipes (Linnaeus, 1767) : H

\section{TETRAGNATHIDAE}

40. Chrysometa conspersa (Bryant, 1945)* : N

41. Chrysometa obscura (Bryant, 1945)* : N, H

42. Chrysometa sp. : N, H

43. Cyrtognatha sp. : N, H

44. Leucauge sp. : N, H

45. Tetragnatha $\mathrm{sp}$ : : $\mathrm{S}$

\section{ARANEIDAE}

46. Acacesia hamata (Hentz, 1847) : H, BT, S

47. Aculepeira busu Levi, 1991* : N

48. Argiope trifasciata (Forsskål, 1775) : N

49. Araneus sp. : N

50. Cyrtophora citricola (Forsskål, 1775) : S

51. Eustala bisetosa Bryant, $1945^{*}: \mathrm{N}$

52. Eustala sp.: $\mathrm{H}$

53. Gea heptagon (Hentz, 1850) : N

54. Metepeira jamaicensis Archer, $1958: \mathrm{N}$

55. Micrathena militaris (Fabricius, 1775) : N, H, BT

56. Neoscona marcanoi Levi, 1993 : N

57. Neoscona sp. : N

58. Ocrepeira serrallesi (Bryant, 1947) : S

59. Parawixia tredecimnotata F. O. P.-Cambridge, 1904 : N, H, BT, S 
60. Wagneriana sp. : H

LYCOSIDAE

61. Hogna sp. : N

OXYOPIDAE

62. Hamataliwa sp. : N, H, S

63. Peucetia viridans (Hentz, 1832) : N, E

CTENIDAE

64. Ctenus sp. : BT

65. Cupiennius sp. : H, BT

AGELENIDAE

66. Barronopsis sp. : BT

MITURGIDAE

67. Syspira sp. : N, H

68. Teminius sp. : N

ANYPHAENIDAE

69. Hibana sp. : BT, S

70. Lupettiana sp. : S

71. Thaloe remotus (Bryant, 1948)* $: \mathrm{H}$

72. Wulfila $\mathrm{sp}$ : : H, E

CLUBIONIDAE

73. Elaver implicata (Gertsch, 1941)* : N

74. Elaver sp. : N, H

TRACHELIDAE

75. Trachelas $\mathrm{sp}$. : $\mathrm{N}, \mathrm{H}$

GNAPHOSIDAE

76. Zelotes sp. : S, E

SELENOPIDAE

77. Selenops pensilis Muma, 1953* : E

78. Selenops sp. : BT, E

SPARASSIDAE

79. Olios $\mathrm{sp}$. : $\mathrm{H}, \mathrm{S}$

80. Stasina saetosa Bryant, 1948* : N, H, S, E

PHILODROMIDAE

81. Apollophanes sp. : H

THOMISIDAE

82. Rejanellus sp. : H

SALTICIDAE

83. Agobardus sp. : N, H

84. Anasaitis sp. : N, BT, E

85. Bythocrotus crypticus Zhang et Maddison, 2012* : E

86. Corythalia minor (Bryant, 1943)* : H

87. Corythalia sp. : N, H

88. Descanso formosus Bryant, 1943* : S

89. Hentzia sp. : BT, S

90. Truncattus $\mathrm{sp} . * / *: \mathrm{N}$

[Recibido: 14 de agosto, 2015. Aceptado para publicación: 15 de septiembre, 2015] 